\title{
Target Detection within Sea Clutter Based on Multifractal Detrended Fluctuation Analysis
}

\author{
Zhan $\mathrm{Xu}^{1, \mathrm{a}}$, Jianwei Wan ${ }^{1, \mathrm{~b}}$, Gang $\mathrm{Li}^{1, \mathrm{c}}$ and Fang $\mathrm{Su}^{1, \mathrm{~d}}$ \\ ${ }^{1}$ School of Electronic Science and Engineering, National University of Defense Technology, \\ Changsha, 410073, People's Republic of China \\ aEmail: xuzhan121@163.com
}

Keywords: Target detection, sea clutter, Multifractal detrended fluctuation analysis.

\begin{abstract}
We present the multifractal detrended fluctuation analysis (MFDFA) for target detection within sea clutter. The multifractal character of the sea clutter time series is discussed. The great hurst parameter differences between sea clutter and target by the detrended fluctuation analysis are available. Experimental results of IPIX datasets show that the proposed method performs better than that based on fluctuation analysis (FA). This type of analysis is promising an efficient framework for analysis of sea radar signals with several potential applications.
\end{abstract}

\section{Introduction}

Target detection within sea clutter is a very important research field in sea remote sensing and radar signal processing. The study of this field may help monitor illegal fishing, and identify marine vessels, navigation buoys, some pieces of spilled oil, etc. Many countries attach more and more importance to the question, and set funds of every sort and kind to support the field. Up to now, a lot of efforts have been made to obtain more efficient methods for target detection within sea clutter. Typical methods include time frequency analysis [1], Hilbert-Huang transform [2], neural network method [3, 4], parameters estimation of Tsallis distribution [5], as well as utilizing the fractal character detecting target $[6,7]$. Fractal method provides an alternative framework to describe the features of the sea clutter [8] and an effective way to detect target [9]. Fluctuation analysis (FA) and detrended fluctuation analysis (DFA) have been applied to characterize the fractal features of sea clutter [9]. These two methods were firstly proposed to analyze the fractal features of DNA sequences and heartbeat time series. Now they have been extensively applied in detecting fractal character for the time series. For ideal fractal processes, FA and DFA yield equivalent results [10]. However, when the data contains some non-stationary trends in practice, DFA often does more reliabley than FA.

Though a great number of works have been appearing, the knowledge about sea clutter is still insufficient. The crux of target detection within sea clutter remains a great challenge. In this paper, we show the multifractal character of the sea clutter time series by the multifractal detrended fluctuation analysis (MFDFA). We use the great hurst parameter differences between sea clutter and target for detecting. Experimental results of IPIX datasets show that the proposed method is more efficient than the method based on FA, which is a good method up to now.

\section{MFDFA Formulation}

In the following, we will present how to construct a "random walk" process and how to estimate the Hurst parameter based on multifractal detrended fluctuation analysis. Let us denote a sea clutter time series by $\boldsymbol{X}=\{\mathrm{X}(i), i=1,2, \ldots, \mathrm{N}\}$. Subtract the mean from $\boldsymbol{X}$, and the above time series changes to $\boldsymbol{x}=\{\mathrm{x}(i), i=1,2, \ldots, \mathrm{N}\}$, where

$$
\mathrm{x}(i)=\mathrm{X}(i)-\sum_{j=1}^{N} \mathrm{X}(j) / N
$$




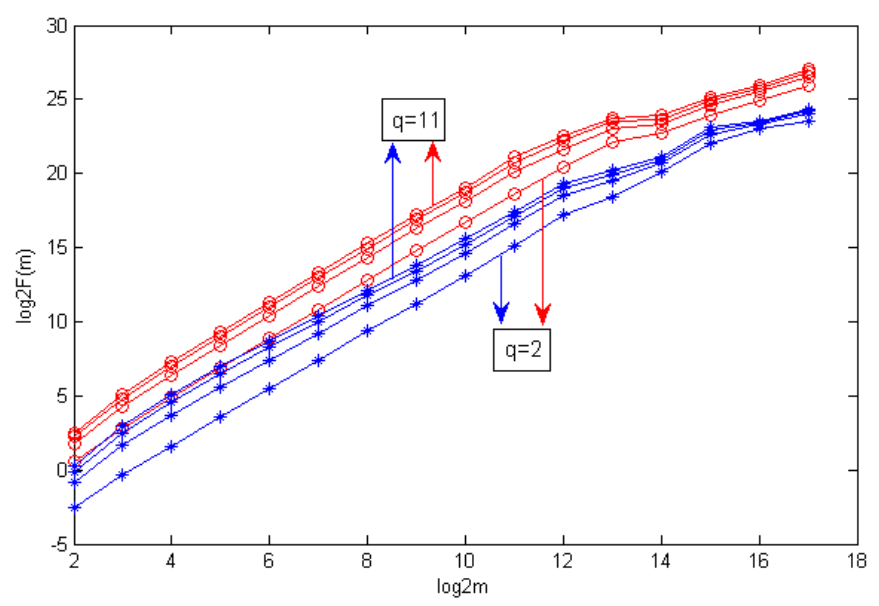

Fig. 1 Representative curves of $\log 2 \mathrm{~F}(\mathrm{~m})$ versus $\log 2 \mathrm{~m}$ for $\mathrm{q}=2 ; 5 ; 8 ; 11$.

Similar to the fractional Gaussian noise process [10], consider the partial summation of $\boldsymbol{x}$ which constructs a new time series $\boldsymbol{y}=\{\mathrm{y}(n), n=1,2, \ldots, N\}$, where

$$
\mathrm{y}(n)=\sum_{i=1}^{n} \mathrm{x}(i) .
$$

Generally, $\boldsymbol{x}$ is called an "increment" process, and $\boldsymbol{y}$ is called a "random walk" process of $\boldsymbol{x}$. MFDFA consists of the following steps [11]. Firstly, divide the given random walk into $[\mathrm{N} / \mathrm{m}]$ nonoverlapping segments with the equal length, where the notation $[\boldsymbol{x}]$ denotes the nearest integer no larger than $\boldsymbol{x}$. Secondly, define the local trend $\mathrm{y}_{l}(n)$ in each segment by the least squares fitting method. In this paper, we use the linear polynomial fitting method for $\mathrm{y}_{l}(n)$. Thirdly, compute the "detrended walk" in each segment, denoted by $\mathrm{y}_{m}(n)$, which is the difference between the original walk $\mathrm{y}(n)$ and the local trend $\mathrm{y}_{l}(n)$. Finally, examine whether the fluctuation function $\mathrm{F}_{\mathrm{d}}^{(\mathrm{q})}(m)$ increases with $m$ in a power law form or not from the following formula

$$
\mathrm{F}_{\mathrm{d}}^{(\mathrm{q})}(m)=\left\langle\sum_{n=1}^{m}\left|\mathrm{y}_{m}(n)\right|^{q}\right\rangle^{1 / q} \sim m^{\mathrm{H}(q)},
$$

where $q$ is real and the angle bracket denotes the ensemble average over all segments. When the power-law regime in (3) exists, it shows that the time series has the fractal character. Furthermore, if $\mathrm{H}(q)$ is not a constant function of $q$, it has the multifractal character; otherwise, it is a monofractal. Obviously, Hurst parameter can be determined from the log-log plot of $\mathrm{F}_{\mathrm{d}}^{(\mathrm{q})}(m)$ versus $\mathrm{m}$ within the range of fractal scales

$$
\mathrm{H}(q)=\log _{2} \mathrm{~F}_{\mathrm{d}}^{(\mathrm{q})}(m) / \log _{2} \mathrm{~m} .
$$

When $q=2$, the formula (3) has the form

$$
\mathrm{F}_{d}(m)=\left\langle\sum_{n=1}^{m}\left|\mathrm{y}_{m}(n)\right|^{2}\right\rangle^{1 / 2} \sim m^{\mathrm{H}} .
$$

The whole analysis process is often called fluctuation analysis (FA) [11].

\section{Data Description}

In this study, the McMaster IPIX datasets are used to be training time series for testing the performances of ESN in detecting target. The McMaster IPIX radar is an instrumentation-quality X-band radar system. The radar data were collected in November 1993 from Osborne Head Gunnery Range (OHGR) at Dartmouth, Nova Scotia, Canada. Fourteen sea clutter measurement datasets were 


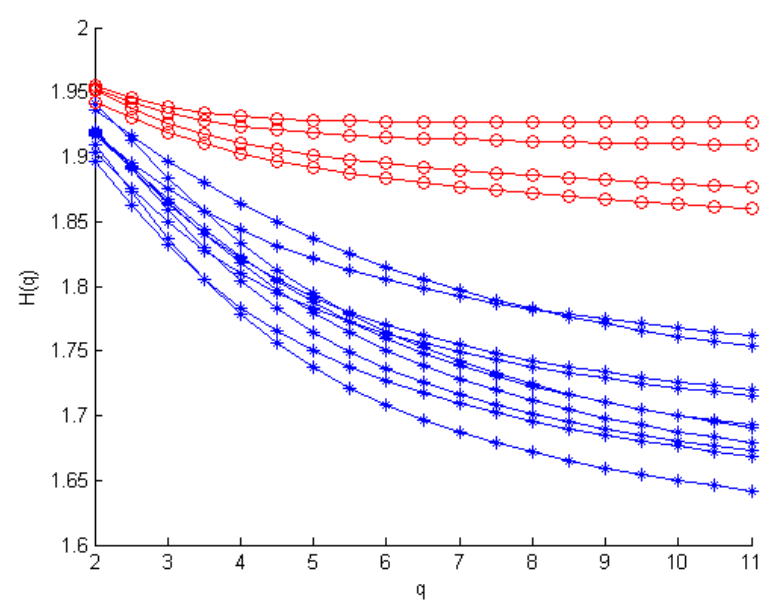

Fig. 2 Variation of $\mathrm{H}(\mathrm{q})$ versus q curves for 14 range bins.

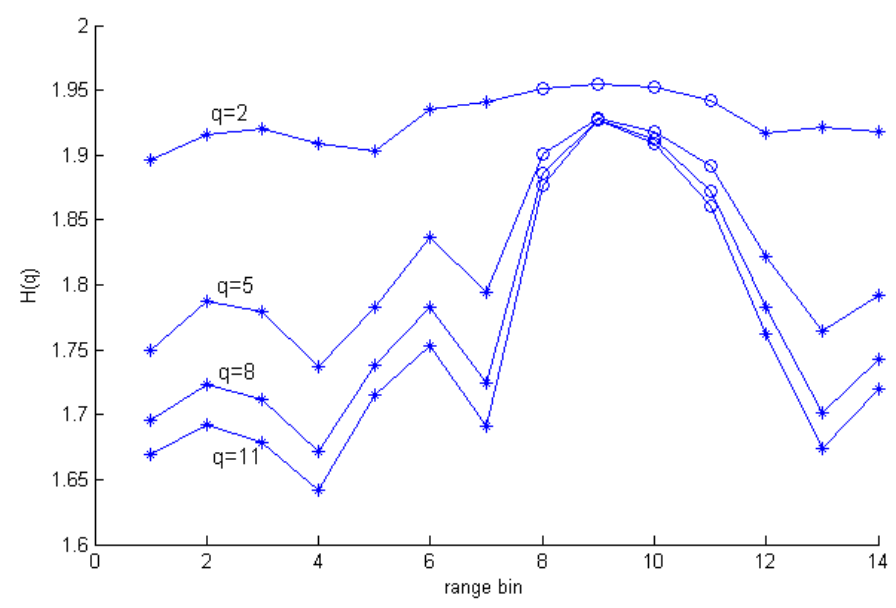

Fig. $3 \mathrm{H}(\mathrm{q})$ values for the 14 range bins, $\mathrm{q}=2 ; 5 ; 8 ; 11$.

obtained from a website maintained by Professor Simon Haykin:

http://soma.ece.mcmaster.ca/ipix/dartmouth/datasets.html. The operating frequency of IPIX radar is $9.39 \mathrm{GHz}$, so the wavelength is about $3 \mathrm{~cm}$. The wave height of the ocean varies from $0.8 \mathrm{~m}$ to $3.8 \mathrm{~m}$, but the peak height even arrives at $5.5 \mathrm{~m}$. The wind conditions vary from 0 to $60 \mathrm{~km} / \mathrm{hr}$ generally, but the gusts reach to $90 \mathrm{~km} / \mathrm{hr}$. The grazing angle varies from less than $1^{\circ}$ to a few degrees. We consider amplitude data of two polarizations, HH (horizontal transmission, horizontal reception) and VV (vertical transmission, vertical reception). Each dataset contains fourteen spatial range bins, and each range bin has $2^{17}$ samples and the sampling frequency is $1000 \mathrm{~Hz}$. The target is a small spherical block of styrofoam wrapped with wire mesh. A few of the range bins hit a target, and the range bin where the target is strongest is labeled as the primary target bin. Due to the target moves around, the bins close to the primary target bin may also hit the target. They are labeled as the secondary target bins.

\section{Experiments}

Use the IPIX radar data, we can get the function $\log _{2} \mathrm{~F}_{\mathrm{d}}^{(\mathrm{q})}(m)$ at different scales $m$ by DFA. Here, the primary target range bin is 9 , and the secondary target range bins are from 8 to 11 . The typical curves of $\log _{2} \mathrm{~F}_{\mathrm{d}}^{(\mathrm{q})}(m)$ versus $\log _{2} m$ for all range bins of sea clutter with different values of $q(q=2,5,8,11)$ are shown in Fig.1. Open circles denote the range bins with the target, while asterisks denote the range bins without the target. It is obvious that all the curves of $\log _{2} \mathrm{~F}_{\mathrm{d}}^{(\mathrm{q})}(m)$ increase with $\log _{2} m$ in a linear manner only in part, which indicates the fractal character of sea clutter relates to the time scale. We observe the curves are fairly linear in the range of $m \in\left(0,2^{12}\right]$. This range corresponds to the time scale range of about $(0 \mathrm{~s}, 4 \mathrm{~s}]$. The sea clutter data in this time scale range have the fractal character. The variations of $\mathrm{H}(q)$ versus $q$ for the fourteen range bins data are shown in Fig.2. It is not difficult to judge that the sea clutter data appear to be multifractal, especially for sea clutter data without the target.

Since the ultimate goal of sea clutter study is to improve the performance of target detection within clutters. Let us examine the effect of MFDFA according to the hurst parameter differences between the target and the sea clutter. The hurst parameter of each curve is calculated by fitting a straight line to the $\log _{2} \mathrm{~F}_{\mathrm{d}}^{(\mathrm{q})}(m)$ versus $\log _{2} m$ curve within the range of $m \in\left(0,2^{12}\right]$. From Fig.3, we notice that the hurst parameters of the curves with the target are much larger than those without the target. As $q$ becomes larger, the difference of $\mathrm{H}(q)$ is more obviously. However, with $q$ becoming more and more larger, the curves have a stable limit. Now let us focus on $q=2$. It is the case based on fluctuation analysis. The detection effectiveness for this case is discussed in [9]. From Fig.3, it is easy to conclude that the target detection based on MFDFA performs better than that based on FA. It turns out that this is a generic feature for all the measurement data. 


\section{Summary}

In summary, we have showed the multifractal character of the sea clutter time series, and introduced MFDFA for detecting target within sea clutter in this paper. We find that sea clutter data are possessed of multifractal character within several seconds, and the multifractal character of pure sea clutter data is more obvious than that with target. According to the difference of the Hurst parameter, the experiments of IPIX data show that target detection by MFDFA is more effective than the case based on FA.

\section{References}

[1] T. Thayaparan and S. Kennedy, Detection of a manoeuvring air target in sea-clutter using joint time-frequency analysis techniques, IEE Proc. Radar, Sonar Navi., 151 (2004) 19-30.

[2] C. Xiao, H. Cha and D. Xia, sea clutter characteristics analysis and target detection based on HHT, Proc Consum. Electron. Comm. Network, (2011) 694-697.

[3] H. Leung, N. Dubash and N. Xie, Detection of small objects in clutter using a GA-RBF neural network, IEEE Tran. Aero. Elec. Sys., 38 (2002) 98-118.

[4] N. Xie, H. Leung and H. Chan, A multiple-model prediction approach for sea clutter modeling, IEEE Tran. Geosci. Remote, 41 (2003) 1491-1502.

[5] V. T. Vakily and M. Vahedi, Sea clutter modeling improvement and target detection by Tsallis Distribution, Int. Conf. Adv. Comput. Control, (2009) 715-719.

[6] X. K. Xu, Low observable targets detection by joint fractal properties of sea clutter: an experimental study of IPIX OHGR datasets, IEEE Trans. Antennas Propag., 58 (2010) 1425-1429.

[7] J. Guan, N. B. Liu, J. Zhang and J. Song, Multifractal correlation characteristic for radar detecting low-observable target in sea clutter, Sign. Proc., 90 (2010) 523-535.

[8] R. A. Schowengerdt, Remote sensing: models and methods for image processing, Aca-demic Press, New York, 2007.

[9] J. Hu, J. B. Gao and F. L. Posner, Target detection within sea clutter: A comparative study by fractal scaling analyses, Fractals, 14 (2006) 187-204.

[10] P. Talkner and R. O. Weber, Power spectrum and detrended uctuation analysis: application to daily temperatures, Phys. Rev. E, 62 (2000) 150-160.

[11] J. B. Gao, Y. H. Cao, W. W. Tung and J. Hu, Multiscale analysis of complex time series, Wiley, New York, 2007. 